ISSN: 2386-3919 - e-ISSN: 2386-3927

DOI: https://doi.org/10.14201/et20163424363

\title{
MUNDOS VIRTUALES. METAANÁLISIS DE EXPERIENCIAS EDUCATIVAS DESDE SUS INICIOS
}

\author{
Virtual worlds. Meta-analysis of educational experiences \\ from its beginnings
}

\author{
Sergio Manuel DíAz FERnÁndeZ \\ Universidad de Murcia \\ Correo institucional: smanuel.diaz@um.es
}

Recibido: 15/10/2015; Aceptado: 25/4/2016; Publicado: 30/11/2016

Ref. Bibl. SERGIO MANUEL DÍAZ FERNÁNDEZ. Mundos virtuales. Metaanálisis de experiencias educativas desde sus inicios. Enseñanza \& Teaching, 34, 2-2016, 43-63.

RESUMEN: Ha pasado más de una década desde que la literatura científica especializada presentara en el año 2004 la primera experiencia educativa con mundos virtuales. Un periodo evolutivo que precisa de análisis para determinar el grado de adecuación de las experiencias desarrolladas hasta el momento, posibilitando la formulación de orientaciones y/o aspectos a considerar en futuras experiencias para alcanzar un mejor ajuste y adecuación al marco específico de trabajo.

La investigación se circunscribe dentro del paradigma metaanalítico, con una revisión cuantitativa y sistemática de los resultados obtenidos en las 36 experiencias a las que se ha tenido acceso, proyectando un análisis y revisión de las mismas mediante la utilización de una ficha de observación, nueva y validada, en la que se recogen una serie de parámetros deseables de cumplimiento, en base al marco teórico actual establecido por diferentes expertos.

Los resultados evidencian un alto grado de adecuación en aspectos didácticos y pedagógicos, así como en comunicación e interacción, siendo menor en aspectos técnicos y de apoyo, lo que puede condicionar el trabajo en mundos virtuales. Por otro lado, se detectan errores al no atender a las características de los participantes. El no tener en cuenta su grado de socialización, sus conocimientos digitales o sus capacidades comunicativas en estos entornos condiciona la validez de los diseños e implementaciones, siendo importante considerarlos en futuras experiencias con mundos virtuales. 
Palabras clave: mundos virtuales; experiencia educativa; enseñanza; aprendizaje; diseño; interacción; comunicación; second life.

SUMMARY: Over a decade has passed since the specialized scientific literature presented in 2004 the first educational experience with virtual worlds. An evolutionary period that requires analysis to determine the adequacy of the developed experiences so far, enabling the formulation of guidelines and / or aspects to consider in future experiences to achieve better adjustment and adaptation to the specific framework.

The investigation lies within the meta-analytic paradigm, with a quantitative and systematic review of the results of the 36 experiences that have been accessed, projecting an analysis and review of the same by using a form of observation, new and validated, in which a number of desirable compliance parameters are set, based on the current framework established by different experts.

The results show a high degree of adaptation in didactic and pedagogical aspects, as well as communication and interaction, being lower in technical and support aspects, which can influence the work in virtual worlds. On the other hand, errors not meet the characteristics of the participants are detected. Failure to take into account their degree of socialization, their digital knowledge or communication skills in these environments, determines the validity of the designs and implementations, being important to consider in future experiences with virtual worlds.

Key words: virtual worlds; educational experience; teaching; learning; design; interaction; communication; second life.

\section{INTRODUCCIÓN}

Las primeras aproximaciones al concepto de mundo virtual aparecen de la mano de Neal Stephenson en el año 1992. La evolución en este campo hace que el término «metaverse» se nutra de avances científicos y tecnológicos que le incitan nuevas características, haciendo que se transforme de un mero espacio tridimensional inmersivo a un amplio concepto que abarca múltiples ámbitos, como es el mundo virtual.

En un primer momento, los videojuegos se erigen como la principal cabeza impulsora de los mundos virtuales, entendiendo los mismos como una serie de espacios dirigidos en los que se desarrollan juegos de rol o simulaciones animadas, con una orientación básica, el ocio y disfrute de sus usuarios. Este marco experiencial permite el surgimiento de nuevos mundos virtuales, destacando en el año 2003 la aparición de Second Life como un hito que permite y facilita el acceso a un grupo más nutrido de personas. Todo ello confiere a los mundos virtuales las posibilidades de uso de nuevos planteamientos y utilidades que permiten ahondar en una amplia diversificación de aplicaciones.

Las evidencias previas permiten afirmar la alta capacidad de interacción y comunicación que ofrecen los mundos virtuales, aspectos que los identifican como 
potentes herramientas de trabajo y gestión del conocimiento, dotando de corporeidad al usuario y estableciendo nexos relacionales mediante espacios dirigidos a múltiples facetas y ámbitos de la sociedad, siendo uno de ellos el educativo. Así, atendiendo a las múltiples posibilidades y desarrollos, se implementan las primeras investigaciones y experiencias orientadas hacia su aplicación educativa al objeto de potenciar los aprendizajes colaborativos, el trabajo activo y la socialización, entre otros aspectos.

La primera constatación de una experiencia educativa desarrollada con mundos virtuales fue llevada a cabo por Warden, Stanworth, Ren y Warden (2013). Synchronous learning best practices: An action research study fue desarrollada entre los años 2004 y 2012, suponiendo un hito importante en este ámbito de trabajo, y al que siguieron otras experiencias similares, especialmente entre los años 2008 y 2009. Es lógico pensar que una de sus principales intenciones es el análisis y estudio de la capacidad de esta herramienta como parte del proceso educativo, bien como elemento principal o como complemento de otros que se utilizan en el aula tradicional.

Por ello, y habiendo transcurrido poco más de una década desde esta primera experiencia educativa con mundos virtuales, este metaanálisis se centra en el análisis de este tipo de experiencias, reflejadas en la literatura científica especializada, desde sus inicios hasta nuestros días. La cuestión de partida de esta investigación es conocer cómo se están realizando estas experiencias educativas, atendiendo para ello a elementos, diseños, metodologías y estrategias que utilizan, revisando los datos extraídos según lo establecido como adecuado por expertos en el campo, para obtener evidencias de aspectos y modificaciones necesarias a considerar, de tal forma que futuras experiencias educativas en mundos virtuales dispongan de una revisión que les permita obtener orientaciones que mejoren sus entornos de trabajo e implementen desarrollos óptimos y de calidad.

El análisis documental ha permitido constatar la existencia de trabajos previos orientados hacia la revisión de estudios de investigaciones empíricas sobre experiencias educativas en estos entornos. En esta línea encontramos estudios como los realizados por Hew y Cheung (2010), Wang y Burton (2012) o Rahman, Yahaya y Halim (2014), que se centran en aspectos descriptivos o de análisis de partes concretas de las experiencias o, en otros casos, nos encontramos con estudios vinculados a un área concreta, como es el caso de los recientes estudios de Miller y Jensen (2014) o Cant y Cooper (2014), centrados de forma exclusiva en la enfermería. Esta situación hace necesaria una revisión de literatura más profunda, amplificando el número de experiencias desarrolladas, y que se encuentran fuera de los marcos de estudios previos, diversificando las áreas, elementos y aspectos de análisis y estudio a considerar, para adquirir nuevas evidencias y conclusiones sobre aquello que pudiera requerir una subsanación, una redefinición o puntualización mediante la relación y cuantificación de los resultados obtenidos. El interés que se deriva de todo ello es la adquisición y comunicación de un conocimiento válido que permita mejorar y adecuar de forma más precisa la utilización de mundos virtuales en este 
ámbito, utilizando el potencial real de los mismos y creando un marco de referencia y análisis para futuros investigadores y diseñadores.

El principal objetivo de esta investigación se centra en el análisis de la literatura científica especializada sobre experiencias educativas con mundos virtuales que aparece en las publicaciones desde el año 2004 hasta principios de 2016. Para ello, se estableció un marco teórico de referencia del que se extraen los parámetros considerados deseables en las mismas por expertos en el campo, para, posteriormente, buscar experiencias educativas de este tipo, analizarlas y determinar su grado de adecuación respecto a esos parámetros deseables.

\section{FUNDAMENTACIÓN SOBRE MUNDOS VIRTUALES}

\subsection{Conceptualización}

Los principios conceptuales de los mundos virtuales se localizan en autores vinculados con la comunidad cyberpunk. De entre ellos, se puede destacar a Gibson (1985), que, en su novela Neuromante, utiliza por primera vez el término ciberespacio. Un término que sufre una clara evolución con el paso del tiempo, hacia lo que llega a considerarse un metaverso, según Stephenson (1992) en su novela Snow Crash.

El metaverso de Stephenson (1992) se conforma como un universo informático que permite una representación de los usuarios mediante avatares que se utilizan como medios de comunicación e interacción en entornos virtuales tridimensionales ubicados en el ciberespacio, y dispuestos de forma similar al mundo real, pero sin las limitaciones físicas propias de éste.

El planteamiento de este autor, unido al auspicio de los avances tecnológicos, supone un punto de inflexión para la creación de los denominados mundos virtuales. Estos son definidos como "la convergencia de 1) una realidad física virtualmente expandida y 2) un espacio físico virtual persistente. Es una fusión de ambos, mientras que los usuarios la experimenten como tal» (Smart et al., 2007: 4). Por su parte, Carr y Pond (2007) los definen como «un mundo paralelo, inmersivo, que simula un universo alternativo, donde habitan simultáneamente miles de personas para comunicarse, jugar y trabajar, en distintos niveles y variantes de juegos de rol con sus avatares» (p. 22).

No obstante, existe una falta de unanimidad conceptual, así como cierta controversia respecto a la diferenciación entre metaverso y mundo virtual. En este sentido, la controversia puede dirimirse en base a las matizaciones establecidas por Stephenson, entendiendo que en el metaverso se genera un intercambio económico, de servicios y/o bienes, una extensión o prolongación del mundo real.

El principal impulso de los mundos virtuales viene sobrellevado por los videojuegos, popularizados por los Multiuser Dungeon (MUD), juegos de rol multiusuario online con interfaces textuales, que acabaron transformándose en los Massively Multiplayer Online Role Playing Games o MMORPG, que, además de gráficos en 3D, 
permitían el desarrollo de espacios para comunidades e interacción entre diferentes usuarios (Varela, 2010).

Con el tiempo, surgen nuevos planteamientos y ámbitos de trabajo y estudio. En este sentido, Kapp (2007) apunta al surgimiento de una nueva orientación hacia el campo educativo, una nueva ruta con la que surgen los Massively Multilearner Online Learning Environment (MMOLE) o Ambientes de Aprendizaje en Línea Masivos para Multiaprendices, cuyo principal objetivo es facilitar y potenciar los procesos de enseñanza y aprendizaje en base a espacios virtuales para múltiples usuarios.

Kapp (2007) destaca la utilidad educativa de los mundos virtuales al permitir el establecimiento de un aprendizaje formal estructurado mediante una simulación prediseñada y de un aprendizaje informal no estructurado mediante procesos de interacción y comunicación, lo que confiere la constitución de un entorno de aprendizaje en el que los usuarios disponen de privilegios a la hora de administrar el aprendizaje, potenciando y generando un aprendizaje más profundo y significativo. Esto hace evidente que los MMLOE se orientan hacia el aprendizaje, la socialización y la capacitación, no creando una consecución lineal de logro de objetivos o puntuaciones, ni una superación de niveles o competición entre rivales, como sí es el caso en los MMORPG. No obstante, de forma puntual, pueden existir juegos en los mundos virtuales, aunque sin constituir un espacio completo de actuación.

Smart et al. (2007) realizaron un estudio prospectivo para la Aceleration Studies Foundation (ASF). En el mismo, se plantean diferentes escenarios agrupados en torno a dos ejes o incertidumbres críticas, lo que permite situar a los mundos virtuales en el segmento simulación-íntimo por ser espacios de simulación que permiten la generación de nuevos espacios y recreaciones de interactuación, centrados en los propios usuarios, en su identidad y en la capacidad de acción de los mismos, conceptualizándolos como herramientas primarias para el aprendizaje.

Muchos autores han profundizado en las características propias de los mundos virtuales. Leask y Younie (2001) establecen que las posibilidades de construcción y manipulación generan procesos más efectivos y motivadores en los usuarios, al tiempo que potencian un rol eminentemente activo al ser ellos mismos los que toman las riendas de su aprendizaje (Huang, Rauch y Liaw, 2010). Mas y Marín (2008) atienden a las posibilidades de proyectar un aprendizaje interactivo, compatible con la docencia de corte tradicional, que permita potenciar la creatividad e innovación desde entornos abiertos e interconectados. Martínez (2009) destaca la importancia de conformar una simulación persistente, disponiendo de una interacción síncrona mediante representaciones identitarias o avatares que potencian procesos de intercambio y creación. Urbina y Flores (2010) se refieren a las posibilidades de obtener información multisensorial, lo que proporciona una mayor aproximación a la realidad. Por su parte, Lizarralde y Huapaya (2012) también se refieren a las posibilidades de navegabilidad que ofrecen entre los diferentes espacios creados y la integración de nuevas aplicaciones, internas o externas al mundo.

Por otro lado, los mundos virtuales disponen de una serie de ventajas específicas. Favorecen la experimentación y el desarrollo de innovaciones con un mayor 
grado de libertad (Kohler, Fueller, Stieger y Matzler, 2010); permiten la realización de ensayos sucesivos, mitigando riesgos y tensiones a la vez que permiten adquirir ciertos grados de experiencia (Arbeláez, 2010); posibilitan nuevas metodologías y procesos de enseñanza y aprendizaje, con rupturas jerárquicas y sin limitaciones geográficas (Grané y Muras, 2006; Mas y Marín, 2008; Galagan, 2008); ofrecen mayor facilidad para la inmersión en el espacio, lo que amplía las oportunidades de conocimiento y exploración (Peachey, 2007); y generan procesos metacognitivos con la práctica como elemento necesario (Cheal, 2007).

Tal y como apunta Warburton (2009), estas y otras características y ventajas no reseñadas propician un cambio en la orientación de los mundos virtuales, pasando de ser meras plataformas recreativas, de ocio y entretenimiento, a espacios de aplicación en el ámbito educativo, que, en un corto espacio de tiempo, disponen de numerosos desarrollos e investigaciones auspiciados por instituciones de índole educativa.

\subsection{Mundos virtuales y educación}

Banet (2001) al hablar de espacios virtuales se refiere a realidades sintéticas que producen una inmersión, un espacio que se va construyendo a medida que se recorre. Por tanto, deben presentarse y desarrollarse estructuradamente, articulados dentro de un sistema coherente, potenciado por actividades y acciones que permitan el desarrollo de tareas de descubrimiento progresivo, lo que va a permitir a los estudiantes establecer una incorporación de los aspectos significativos de las acciones que desarrollan a su estructura cognoscitiva (Ausubel, Novak, Hanesian, Sandoval y Botero, 1976).

Así, el trabajo educativo en mundos virtuales favorece el surgimiento de proposiciones en los estudiantes que les permite avanzar en sus descubrimientos, evolucionándolos y modificándolos a medida que se profundiza, acrecentando el aprendizaje colaborativo y exploratorio en un constante desarrollo resolutivo. En esta línea, Sherman y Judkins (1994) entienden que uno de los principales desarrollos académicos estaría en la formación en facultades de medicina, mientras que García Ruiz (1998) se refiere a sus posibilidades en campos como la arquitectura y los entrenamientos de tipo técnico.

No obstante, Ríos y Ruiz (2011) explicitan que para su utilización en el contexto educativo es preciso considerar extremos como son la planificación de tiempos, actividades y recursos, un diseño ajustado a la realidad y establecer una orientación mayormente práctica que busque el desarrollo integral de la persona. Esto precisa del establecimiento de momentos de observación y reflexión sobre criterios de integración, tales como los destinatarios de la acción a desarrollar, el contexto sociocultural y escolar y las variables curriculares y del propio medio de trabajo (Martínez, 2007).

Rutherfoord y Rutherfoord (2007) trabajaron sobre los aspectos instruccionales de los mundos virtuales. Sus estudios dieron como resultado una propuesta sobre 
la aplicación del Diseño Instruccional Universal en base a siete principios que se deben considerar a la hora de diseñar un mundo virtual: 1) Uso accesible y justo, 2) Flexibilidad en su utilización, participación y presentación, 3) Sencillez y coherencia, 4) La información se presenta de forma explícita, percibiéndose fácilmente, 5) Apoyo al estudiante, 6) Minimizar o eliminar esfuerzos o requerimientos innecesarios y 7) Espacio adaptable.

Se hace evidente que para el desarrollo de experiencias educativas con mundos virtuales se deben atender y respetar múltiples consideraciones y requerimientos, confiriendo una gran complejidad que precisa de la coordinación de un equipo multidisciplinar. Al menos, un experto disciplinar, un experto pedagogo, un modelador 3D, un programador y un encargado de la plataforma (Varela, 2010).

Como se indicó, la simulación es muy importante en este tipo de experiencias, dado que se vincula de forma directa con las posibilidades de experimentación, y ésta con la inmersión y la interacción social aparejada (Urbina y Flores, 2010). Pero estos aspectos no son más importantes que la implementación de reevaluaciones sobre los desarrollos utilizados (Mas y Marín, 2008), con el fin de reformular e introducir modificaciones que mejoren las experiencias y les confiera un mayor ajuste, calidad y eficacia educativa.

En este sentido, es importante atender a los planteamientos establecidos por Sangrà (2001), Duart (2003) y Cabero (2006), entendiendo la importancia de establecer parámetros organizativos sobre la información vinculada con elementos del ámbito tecnológico, administrativo y académico, que no solo posibilitará un adecuado uso de la misma en la experiencia educativa, sino que también permitirá proyectar análisis eficaces y válidos sobre la formación real que se realiza en estos ambientes virtuales.

Llegados a este punto, es importante destacar el papel y la importancia de Second Life en el ámbito educativo, siendo un ambiente de inmersión en el que están representadas la mayor parte de las Universidades a nivel mundial (Poveda y Thous, 2013). Livingstone y Kemp (2006) señalan que dispone de una interfaz más atractiva y versátil, mientras que Castronova (2001) resalta su interactividad, corporeidad y persistencia. Sea como fuere, la realidad es que supone un referente en cuanto a mundos virtuales, con ciertas limitaciones a consecuencia de lags o cacofonías. Puede ser que el mayor éxito de Second Life se deba a que «la experiencia afectiva que los estudiantes disfrutan en estos no tradicionales e informales ambientes de aprendizaje no se puede comparar a las plataformas cMs actuales" (Livingstone y Kemp, 2006: 1).

No obstante, la explicación sobre la expansión que han tenido los mundos virtuales en el contexto educativo también se puede encontrar en las amplias posibilidades de crearse, de aprender a ser uno mismo, de construir, de socializarse e interactuar sin limitaciones físicas, así como por las posibilidades del desarrollo de un aprendizaje experiencial, implementado por las posibilidades de recrear laboratorios, simulaciones y espacios educativos seguros y accesibles (Carr, 2008). 


\section{Metodología y PROCEDimiento}

Teniendo en cuenta estos objetivos, se asumió un paradigma metaanalítico, que va a permitir la integración y la revisión, cuantitativa y sistemática, de los resultados obtenidos, para obtener evidencias empíricas sobre la adecuación y concreción de dichas investigaciones (Sánchez y Alto, 1989).

La metodología seguida se define en base a las etapas establecidas por McMillan y Schumacher (2009) y por la formulación y secuencias determinadas por Cooper (1989) y Rosenthal (1991). En un primer momento, se acotaron los criterios de búsqueda mediante la utilización de términos clave. Para la búsqueda, se utilizó la Web of Science (wos) al ser una base de datos de reconocido prestigio, fiable y que permite el acceso a referencias bibliográficas de carácter científico y tecnológico a nivel mundial. Los resultados iniciales fueron:

TABLA 1

Criterios de búsqueda de experiencias y sus resultados

\begin{tabular}{|c|c|}
\hline CRITERIOS DE BÚSQUEDA & RESULTADOS \\
\hline «Mundo virtual» & 24 registros \\
\hline «Mundos virtuales» & 10 registros \\
\hline «virtual world» & 5.179 \\
\hline «virtual worlds» & 2.828 \\
\hline «virtual world» teaching & 245 \\
\hline «virtual worlds» teaching & 279 \\
\hline «Mundo virtual» enseñanza & 0 \\
\hline «Mundos virtuales» enseñanza & 1 \\
\hline «virtual world» education & 531 \\
\hline «virtual worlds» education & 533 \\
\hline «Mundo virtual» educación & 3 \\
\hline «Mundos virtuales» educación & 1 \\
\hline «virtual world» teaching and education & 129 resultados totales \\
\hline «virtual worlds» teaching and education & 175 resultados totales \\
\hline «Mundo virtual» enseñanza y educación & 0 \\
\hline «mundos virtuales» enseñanza y educación & 0 \\
\hline
\end{tabular}

Por la amplitud de los resultados se realizaron sucesivas acotaciones, aproximándonos a un entorno más realista y ajustado de análisis utilizando los 304 resultados extraídos de los criterios "virtual world» teaching and education y "virtual worlds" teaching and education. Estos registros fueron analizados, descartando aquellos que no eran experiencias educativas en mundos virtuales, las duplicidades y algunas a las 
que no se disponía de acceso, quedando la muestra conformada en 36 experiencias. Todas ellas han sido revisadas desde fuentes primarias, lo que permite disponer de una mayor cantidad de detalles para el análisis (McMillan y Schumacher, 2009).

En este punto, y ante la ausencia de una herramienta válida y fiable para los objetivos definidos, se desarrolla un instrumento de recogida ${ }^{1}$ de información mediante las aportaciones de expertos, todas ellas extraídas de una profunda revisión del marco teórico vinculante, y que se acaba concretando en una ficha compuesta por 95 ítems en la que se recogen los principales parámetros que son deseables en el desarrollo de una experiencia educativa en mundos virtuales (Díaz Fernández, 2014), utilizando una escala dicotómica al entender que es la más adecuada, al poder constatar o inferir solo el cumplimiento de los ítems y no su intensidad. Los ítems se agrupan en torno a tres grandes bloques o categorías de análisis y sus secciones correspondientes: aspectos técnicos y de apoyo (a nivel general, usabilidad, diseño de recursos y equipo multidisciplinar), comunicación e interacción y aspectos didácticos y pedagógicos (consideraciones iniciales, diseño instruccional, aspectos previos a la implementación, implementación de la experiencia y evaluación y seguimiento).

Al ser una herramienta de nueva creación, se realizó una validación por juicio de expertos ante la necesidad de consensuar juicios sobre los ítems (Latiesa, 2003), precisando la intervención de diferentes expertos que revisaron, analizaron y valoraron la misma (Wiersma, 2001), lo que se realizó atendiendo a criterios de redacción, de univocidad, de pertinencia y de importancia de todos los ítems. De este proceso resultaron diferentes modificaciones y la eliminación de algunos ítems, pasando de los 117 iniciales a los ya indicados.

Entendiendo las limitaciones de una revisión documental de este tipo, la aplicación de la ficha de observación se realizó mediante la utilización de una escala dicotómica, dada la imposibilidad en muchos casos de obtener una valoración de los distintos niveles de adecuación, tan solo pudiendo constatar la asunción de los parámetros deseables.

\section{Resultados OBTENidos}

A nivel general, se puede observar como las experiencias se desarrollan en ciclos educativos formales, en su mayoría niveles educativos superiores o universitarios (89\%), solo disponiendo de dos en niveles de secundaria, una en primaria y una en educación especial. De entre estas experiencias, el 53\% se realizan con muestras de tamaño inferior a los 60 participantes, y solo el 31\% dispone de muestras grandes, superiores a los 100 participantes.

1. Para una información más detallada de la ficha de observación ver: Díaz Fernández (2014). 
Las materias más abordadas en las experiencias educativas se centran en procesos vinculados con la educación (8 experiencias), la ingeniería y el medio ambiente ( 5 cada una), la medicina y la enfermería ( 4 y 2 experiencias respectivamente). De ellas, el 92\% se desarrollan en centros universitarios, cuya localización principal se encuentra en EE. UU. (25\%), en España y Australia (14\%). Destacando otros desarrollos realizados en la Unión Europea como en como Grecia (8,33\%), Reino Unido y Finlandia (5,55\%). El mundo virtual más elegido es Second Life $(58,34 \%)$, destacando que en algunas se generan mundos específicos diseñados para sus propias experiencias, y en los últimos años algunas han abogado por la utilización conjunta de dobles plataformas, Second Life y OpenSim.

La etapa de mayor auge se localiza entre los años 2008 y 2009, con 13 experiencias educativas. Desde el año 2006 se observa un crecimiento de estas experiencias, siendo el año 2009 en el que generan mayor número. Por otro lado, los datos evidencian un mayor número en el periodo de 2010 a 2014, con 19 experiencias.

Atendiendo a los datos de la Tabla 1, se puede afirmar que en la mayoría de los casos se cumple con los parámetros deseables del marco teórico de referencia. Concretando, 6 experiencias alcanzan niveles de adecuación superiores al 85\% y 15 se distribuyen en cotas del 85\% al 70\%. Es importante destacar que solo 1 de estas experiencias presenta muy bajos niveles de adecuación, apenas alcanzando el 50\% de los parámetros.

En este punto, es necesario matizar que, al tratarse de un análisis documental de experiencias, algunos aspectos no se han podido constatar o inferirse de los textos analizados. En 14 de las experiencias no se han podido determinar algo más del $15 \%$ de los ítems, mientras que, por el contrario, en 14, las limitaciones son inferiores al $11 \%$, tal y como se refleja en la Tabla 1.

TABLA 2

Datos sobre el grado de adecuación de las experiencias

\begin{tabular}{|c|c|c|c|}
\hline EXPERIENCIA & SÍ & NO & N/C \\
\hline 1 & 82,1 & 6,32 & 11,58 \\
\hline 2 & 90,52 & 5,26 & 4,21 \\
\hline 3 & 75,79 & 4,21 & 20 \\
\hline 4 & 82,11 & 4,21 & 13,68 \\
\hline 5 & 66,32 & 12,63 & 21,05 \\
\hline 6 & 78,95 & 7,37 & 13,68 \\
\hline 7 & 70,53 & 7,37 & 22,11 \\
\hline 8 & 62,11 & 13,68 & 24,21 \\
\hline 9 & 73,68 & 5,26 & 21,05 \\
\hline 10 & 82,11 & 0 & 17,89 \\
\hline 11 & 93,68 & 0 & 6,32 \\
\hline
\end{tabular}




\begin{tabular}{|c|c|c|c|}
\hline EXPERIENCIA & SÍ & NO & $\mathrm{N} / \mathrm{C}$ \\
\hline 12 & 64,21 & 11,58 & 24,21 \\
\hline 13 & 82,11 & 2,11 & 15,79 \\
\hline 14 & 52,63 & 32,63 & 14,74 \\
\hline 15 & 54,74 & 26,32 & 18,95 \\
\hline 16 & 64,21 & 28,42 & 7,37 \\
\hline 17 & 76,84 & 15,79 & 7,37 \\
\hline 18 & 87,37 & 8,42 & 4,21 \\
\hline 19 & 51,58 & 40 & 8,42 \\
\hline 20 & 92,63 & 5,26 & 2,11 \\
\hline 21 & 89,47 & 7,37 & 3,16 \\
\hline 22 & 74,74 & 14,74 & 10,53 \\
\hline 23 & 68,42 & 14,74 & 16,84 \\
\hline 24 & 61,05 & 21,05 & 17,89 \\
\hline 25 & 55,79 & 16,84 & 27,36 \\
\hline 26 & 78,95 & 11,57 & 9,47 \\
\hline 27 & 83,15 & 6,31 & 10,52 \\
\hline 28 & 55,79 & 33,68 & 10,52 \\
\hline 29 & 57,89 & 29,47 & 12,63 \\
\hline 30 & 53,68 & 13,68 & 32,63 \\
\hline 31 & 51,58 & 29,47 & 18,94 \\
\hline 32 & 34,73 & 33,68 & 31,57 \\
\hline 33 & 77,89 & 14,73 & 7,36 \\
\hline 34 & 86,31 & 8,42 & 5,26 \\
\hline 35 & 81,05 & 6,31 & 12,63 \\
\hline 36 & 77,89 & 8,42 & 13,68 \\
\hline
\end{tabular}

Proyectando el análisis por bloques y secciones, según los datos de la Tabla 2 , en el bloque de aspectos técnicos y de apoyo se presenta a nivel general una escasa adecuación del 47,78\%, siendo más acuciante en la utilización de prims u objetos reciclados, así como en la utilización de mundos virtuales Open Source (32\% y 26\%, respectivamente). En el lado opuesto, se localiza una alta adecuación sobre la estabilidad del mundo y la persistencia del mismo, llegando a cotas del $84 \%$ y del $82 \%$ respectivamente. En la usabilidad se mejora algo el grado de adecuación, poco más del 57\%, destacando como la adecuación más baja la presencia de cacofonías (59\%) y como la más alta, la utilización de mensajes claros (94\%). 
En el diseño de recursos, se localizan las cotas más altas de adecuación del bloque $(86,12 \%)$, disponiendo en la mayoría de sus ítems de valores superiores al $90 \%$, utilizando recursos específicos orientados hacia la experiencia en todos los casos, aunque el ser copia fiel del entorno real solo se presenta en un 70\% de los casos. Por su parte, en todos los casos constatados, se refleja la importancia y necesidad de un equipo multidisciplinar de trabajo.

Respecto a la comunicación e interacción, su adecuación es del 72,75\%, a pesar de su importancia en la utilización de mundos virtuales. Presenta un nivel de adecuación superior al 85\% en parámetros como dotar a la experiencia de una adecuada comunicación entre participantes, compartir sus conocimientos y posturas, ofrecer una interacción total con el entorno virtual y la presencia de un acompañamiento de docentes y/o expertos. En el lado opuesto, se encuentran parámetros deseables como el establecimiento de una socialización previa o la interconexión entre mundos virtuales ( $47 \%$ y $37 \%$ respectivamente).

En el bloque de aspectos didácticos y pedagógicos el grado de adecuación general es del $76 \%$, siendo el bloque que engloba mayor cantidad de ítems, presentando especial relevancia en secciones sobre diseño instruccional y evaluación y seguimiento, en las que se alcanzan cotas de adecuación del $84 \%$ y del $83,74 \%$ respectivamente. La nota discordante surge en la sección de consideraciones iniciales, solo alcanzado una adecuación del 60,7\%.

En la sección de consideraciones iniciales es destacable la completa adecuación de todas las experiencias respecto a la planificación didáctica previa y a su adecuación al currículo, disponiendo de un alto grado de adecuación en considerar las características propias de los participantes y en su conocimiento de la simbología específica ( $83 \%$ y 94\% cada una), aunque se desvían en otros aspectos como la necesidad de estudiar sus conocimientos sobre mundos virtuales (31\%), sus competencias comunicativas (26\%) y el grado de socialización de los estudiantes (17,64\%).

Por un lado, en los parámetros sobre diseño instruccional, se presenta bajo grado de adecuación en la adaptación a los diferentes estilos de aprendizaje (50\%), siendo muy superior en la accesibilidad y en la presencia de información sin ambigüedades (en torno al 97\% cada una) y casi total en el resto de ítems. Por otro lado, en la sección de aspectos previos a la implementación, se identifica una adecuación superior al 78\%, resaltando que en todas las experiencias el aprendizaje está centrado en los participantes. No obstante, la adecuación es menor en aspectos como en la utilización de distintos tipos de agrupamientos (61\%) y en la realización de sesiones de prueba o pruebas piloto (60\%).

En lo referente a la sección sobre implementación de la experiencia, en general solo se alcanzan cotas de adecuación del $73 \%$, ofreciendo un alto cumplimiento en parámetros como el no transmitir solo contenidos o en potenciar la participación activa (97\% cada una). Los parámetros más débiles se localizan en la posibilidad de generar espacios nuevos en el mundo virtual por cualquier participante (31\%) y en la proyección de adaptaciones a medida que se desarrollan las experiencias (24\%). 
Por último, sobre la evaluación y seguimiento se puede afirmar que presenta unas cotas altas, donde la mayoría de los parámetros se encuentran entre el 85\% y el 95\%. Sólo se encuentran por debajo la existencia de una retroalimentación (80\%) y la realización de análisis sobre los métodos utilizados (76\%).

TABLA 3

Datos sobre el grado de adecuación por bloque

\begin{tabular}{|c|c|c|c|}
\hline & Sí & NO & $\mathrm{N} / \mathrm{C}$ \\
\hline \multicolumn{4}{|c|}{ ASPECTOS TÉCNICOS Y DE APOYO } \\
\hline A nivel general & 47,78 & 24,17 & 28,05 \\
\hline Usabilidad & 57,23 & 9,44 & 33,33 \\
\hline Diseño de recursos & 86,12 & 7,63 & 6,25 \\
\hline Equipo Multidisciplinar & 72,69 & 13,89 & 13,42 \\
\hline \multicolumn{4}{|c|}{ COMUNICACIÓN E INTERACCIÓN } \\
\hline & 72,75 & 14,23 & 13,02 \\
\hline \multicolumn{4}{|c|}{ ASPECTOS DIDÁCTICOS Y PEDAGÓGICOS } \\
\hline Consideraciones iniciales & 60,69 & 20,94 & 18,37 \\
\hline Diseño instruccional & 84,03 & 10,19 & 5,78 \\
\hline Aspectos previos & 78,48 & 13,42 & 8,1 \\
\hline Implementación & 73,05 & 20,55 & 6,4 \\
\hline Evaluación y seguimiento & 83,74 & 9,12 & 7,14 \\
\hline
\end{tabular}

\section{CONCLUSIONES}

Las universidades se erigen como los centros que más esfuerzos han aunado para realizar este tipo de experiencias. La mayor parte se diseñan y dirigen desde estos centros, manteniendo una presencia simbólica en otros niveles educativos. La implementación de experiencias en niveles educativos superiores puede constituir una buena herramienta de trabajo al disponer en gran medida de participantes con conocimientos informáticos, con experiencias y aproximaciones a las TIC más amplias que en otros niveles, lo que podría explicar grosso modo esta direccionalidad educativa. No obstante, es lógico pensar que se podría alcanzar una mayor profundidad si se proyectan en niveles de primaria, de secundaria o de atención a necesidades educativas especiales. Entendiendo que la línea de trabajo debería coadjuntar a todos los niveles, posibilitando un mayor conocimiento.

Esa orientación podría ser la causa de que no se profundice en conocer los aspectos formativos básicos de los participantes, al entender que disponen de los mismos y que tienen un grado de socialización adecuado para este trabajo. Una posible equiparación según el nivel formativo alcanzado por los participantes 
que implicaría una concepción errónea de un perfil único universitario, precisando de un proceso indagatorio, que no supondría una gran amplitud de trabajo, pero que reportaría importantes beneficios al alcanzar una adecuada comprensión de la diversidad con la que está trabajando, sus requerimientos propios y adaptaciones.

Es evidente que no atender de forma más profunda a las características y particularidades del alumnado participante es una forma de obrar que atenta contra principios básicos de cualquier marco educativo. La divergencia y la diversidad deben ser atendidas de forma escrupulosa, adecuándose a los patrones educativos actuales y a los parámetros ya establecidos al inicio de este metaanálisis. Por ende, no conocer de forma concreta el nivel de conocimientos, las capacidades comunicativas o el grado de socialización se puede considerar como un error grave que perjudica a cualquier proyecto educativo de este tipo.

Otro aspecto destacable de muchas de las experiencias es el reducido tamaño de las mismas. Es lógico pensar que la complejidad y el coste de este tipo de proyectos limitan el espacio y la cantidad de participantes, pero también es importante atender a que la utilidad de muestras tan reducidas limita mucho la capacidad de obtener resultados y conclusiones fiables, al tiempo que se hace imposible reforzar la validez y la generalización de cualquier dato extraíble. Hay que considerar que el trabajo debe orientarse hacia la obtención de un conocimiento más profundo que permita conformar cauces y parámetros de actuación, que refuercen la utilidad de estos entornos virtuales en la formación y facilite el desarrollo de futuros trabajos.

Estas experiencias, apoyadas muchas veces en Second Life, se sitúan en localizaciones puntuales. Destacan Estados Unidos, España y Australia, lo que puede servir como punto de revisión y análisis ante futuras experiencias. En general, sus parámetros de trabajo y actuación presentan altos índices de éxito y adecuación a los marcos teóricos actuales, lo que nos hace entender el alto grado de conocimiento sobre las peculiaridades y requerimientos del trabajo inmersivo en mundos virtuales.

Es relevante observar que las experiencias no se dirigen solo a enseñar contenidos, sino que presentan en muchos casos una carga empática, de aprendizaje de comportamientos saludables y de valores de convivencia y trabajo. Una característica que supone el establecimiento de un proceso educativo más complejo, poniendo en juego un aprendizaje multidireccional que puede permitir alcanzar altos niveles de formación en los estudiantes. Las diversas incursiones en campos como el medio ambiente, la medicina o la enfermería reforzarían esta conclusión, entendiendo que no se pretende una adquisición de conocimientos, sino la adquisición de habilidades y actitudes que complementen y acaben formando a los estudiantes de una forma más integra y preparada para afrontar los retos sociales y laborales que les esperan.

La utilización de mundos virtuales con fines educativos implica altos requerimientos técnicos y de apoyo a los que subyacen todas las experiencias y proyectan ajustes y concreciones adecuadas, analizan las características de los entornos virtuales y atienden a la necesidad de un trabajo multidisciplinar, coordinado y complejo. 
No obstante, no se debe olvidar que se trata de una experiencia educativa. Con ello, se pretende puntualizar que los requerimientos pedagógicos y didácticos son sumamente importantes y deben ser tratados con mucho cuidado y análisis.

Dependiendo del momento y de las necesidades, son muchos los pormenores a considerar que precisan de revisión y reconfiguración. Así, la ausencia de sesiones de pruebas para realizar una valoración inicial y previa a la implementación es desconcertante, máxime cuando se hace evidente que en todo nuevo desarrollo se soslayan aspectos y se desconocen otros. Una puesta a punto en este sentido es ineludible si la intención es alcanzar cotas de formación aceptables y potenciar entornos viables de trabajo.

Se puede afirmar que en la implementación de las experiencias se proyecta un trabajo activo, que causa motivación en los estudiantes y pone a prueba en gran medida sus habilidades. Esto hace que los entornos de trabajo creados permitan una mayor implicación e integración de todos los participantes en las actividades educativas, alcanzando amplios logros y potenciando la inmersión de los mismos. Aunque como aspecto negativo destaca la falta de adaptaciones a medida que se desarrollan las experiencias. En general, no se proyectan cambios o modificaciones en este punto, lo que puede ser contraproducente y limita las posibilidades educativas de la experiencias desarrollada.

Dentro de los mencionados siete principios de Rutherfoord y Rutherfoord (2007), se establece que los espacios deben ser adaptables, circunscritos a los perfiles de los participantes como elemento clave del éxito de las experiencias. Las adaptaciones son propias de cualquier formación. En cambio, parece que en algunas experiencias los expertos se dejan embriagar por el entorno tan rico en el que están trabajando y menguan sus esfuerzos hacia la conceptualización básica de los procesos de enseñanza y aprendizaje.

También sería importante reflexionar sobre el enfoque de aislamiento que presentan la mayoría de las experiencias en mundos virtuales. No suele existir una interconexión entre mundos, y el intercambio o la utilización de objetos o prims reciclados es muy limitada. En este sentido, se debería replantear el establecimiento de los cauces colaborativos, en especial, en ciclos superiores de formación. Partir de una experiencia desde cero supone mucho trabajo y un gran coste, pero, si al igual que sucede en otros procesos, se proyectaran líneas de trabajo cooperativo, de intercambio de elementos y de comunicación entre líneas investigadoras, se posibilitaría un incremento de posibles experiencias, de ampliar el campo de conocimiento práctico y de la consecución de adecuaciones educativas óptimas.

Viendo los datos de los últimos cinco años, se pueden constatar mejoras, en especial respecto a la presencia de manuales o guías de apoyo, el análisis de los métodos utilizados, la presencia de retroalimentación y la reducción de lags prolongados, entre otros. Aunque se acrecienta la inobservancia sobre el conocimiento de las características de los estudiantes y de sus necesidades de adaptación, lo que precisaría del establecimiento de un foco de atención hacia futuras experiencias, debiendo incrementar su cuidado en los desarrollos que se proyecten. 


\section{DisCUSIÓN}

En los últimos años se ha evidenciado un alto grado de madurez de los mundos virtuales. En este grupo destaca Second Life, consolidándose como un referente de los mundos virtuales. Su utilización como principal entorno de trabajo ha sido referido en diversos estudios anteriores (Inman, Wright y Hartman, 2010; Rahman, Yahaya y Halim, 2014). No obstante, la prospectiva según los datos de los últimos cinco años erige a este mundo virtual más allá de lo establecido anteriormente, y se sitúa como el preferido en aplicaciones de ámbito educativo dejando relegados a otros como OpenSim.

La revisión realizada por Wang y Burton (2012) establece un continuo temporal según publicaciones sobre mundos virtuales. Los datos recabados se correlacionan con ese continuo, confirmando el año 2009 como el punto más álgido en este campo. Si bien, se puede confirmar que posteriormente se produce un serio decrecimiento, posible fruto de la crisis mundial y los grandes costos de implementación, aunque los mundos virtuales vuelven a retomar fuerza en el año 2011, manteniendo un amplio desarrollo en los últimos cinco años con 19 experiencias constatadas.

La evolución de los mundos virtuales también ha influido en su orientación de trabajo. Hew y Cheung (2008) se referían a desarrollos en ramas politécnicas, mientras que Wang y Burton (2012) ya se referían más a orientaciones de formación y negocios. La evidencia nos sitúa en un plano intermedio de ambos estudios. Destacan los desarrollos de formación docente, aunque también se continúa con trabajos en el ámbito de la ingeniería, el medio ambiente y la salud. Aspectos que permiten afirmar la utilidad e incursión de los mundos virtuales en múltiples áreas y ámbitos de trabajo.

Sobre las muestras, hay dos aspectos importantes a considerar. Por un lado, el tamaño de las mismas suele ser reducido. Si bien, Inman, Wright y Hartman (2010) ya atendían a esta circunstancia y hacían evidente la necesidad de acrecentar las mismas. Finalizada esta investigación, poco han cambiado las mismas, trabajando en ocasiones con grupos mínimos que poco pueden aportar al corpus del conocimiento sobre mundos virtuales. Por otro, la necesidad de aprovechar el crecimiento y madurez de los mundos virtuales para implementar experiencias educativas en niveles no universitarios. Una circunstancia ya referida en estudios previos (Hew y Cheun, 2008; Inman, Wright y Hartman, 2010; Wang y Burton, 2012) y que sería necesaria para alcanzar cotas más profundas de conocimiento y comprensión.

En las experiencias educativas en mundos virtuales a las que se ha tenido acceso, se observa que a nivel general se da una alta adecuación a parámetros teóricos establecidos actualmente. Un aspecto que, aunque no se ha podido contrastar con estudios similares, permite evidenciar la buena dirección en el trabajo en estos entornos. Esto no quita que en algunos casos sea necesaria la asunción de determinados cambios para lograr una mayor tasa de adecuación y éxito, si bien, los desarrollos suelen estar orientados hacia las necesidades y peculiaridades que un entorno de este tipo requiere. 
Se hace evidente que en un pequeño porcentaje de las mismas hay inobservancia de múltiples parámetros deseables de cumplimiento. Con ello, nos referimos a la escasa consideración y tratamiento de aspectos técnicos y de apoyo, como la continuidad y vinculación con una LMS. A su vez, se presentan problemas de usabilidad a consecuencia de lags largos y cacofonías o la dificultad de maniobrar con el avatar por el entorno virtual. Los problemas técnicos ya estaban referidos por Hew y Cheung (2008) y más recientemente por Rahman, Yahaya y Halim (2014), lo que debería ser foco de interés previo a la experiencia. En este sentido, al igual que ya plantearon Inman, Wright y Hartman (2010), sería recomendable el establecimiento de un proceso investigativo sobre los requisitos y requerimientos técnicos necesarios.

Por otro lado, se asume y proyecta de forma clara la necesidad de disponer de un equipo multidisciplinar de expertos que apoyen la experiencia, así como del diseño de recursos específicos para estos entornos, y la concreción de espacios que faciliten y permitan las interacciones entre todos los participantes. Los datos constatables sobre usabilidad nos permiten afirmar que se tienen en gran consideración, en especial, respecto a la aparición de distractores. Un aspecto que va a permitir una mayor y mejor inmersión de los participantes en la experiencia desarrollada, registrando una mejora con respecto a las conclusiones establecidas por Inman, Wright y Hartman (2010).

En cambio, en el lado opuesto, las consideraciones generales adolecen de un escaso interés y estudio en gran parte de ellas. Aunque hablamos de marcados aspectos del entorno virtual, su falta de atención afecta y condiciona a desarrollos posteriores llevados a cabo. Es imprescindible atender a la necesidad de disponer y facilitar el acceso a manuales y guías de apoyo, así como permitir y facilitar la ejecución de aplicaciones externas al entorno virtual como elementos que no limiten ni restrinjan la capacidad y habilidades de indagación y aprendizaje de los alumnos, dotándolos de más herramientas para su trabajo virtual, al tiempo que reducirán la ansiedad y proyectarán las experiencias con más posibilidades de éxito.

Sin lugar a dudas, la comunicación y la interacción son aspectos sumamente importantes en estas experiencias. En general, se permite y facilita la comunicación entre estudiantes y docentes desde un mismo nivel jerárquico, orientando las actividades a compartir y presentar productos, desarrollando altos niveles de interactividad con el entorno. Lo que no parece comprensible es la ausencia de momentos de socialización previa a la implementación, lo que explicaría las dificultades de relacionarse con los participantes y el entorno que explicitan Rahman, Yahaya y Halim (2014). Por ello, sería perceptivo y necesario el establecimiento de periodos de socialización para establecer lazos y nexos de intercambio y evitar la falta de participación efectiva, la desorientación o el aislamiento en determinados momentos, suponiendo un enlentecimiento o retrancamiento de la experiencia.

La situación anterior puede agravarse si no se atienden y conocen de forma específica las características de los participantes, que suele darse en gran medida. Hew y Cheung (2008) e Inman, Wright y Hartman (2010) reconocían como 
hándicap la falta de familiaridad con el entorno de trabajo. Es comprensible que la falta de familiaridad con el entorno genere situaciones de rechazo que ponen en peligro el desarrollo de las experiencias, lo que debería suponer un punto de reflexión a la hora de plantear experiencias futuras con mundos virtuales. Conocer el punto de partida de todos los participantes implicados debe instaurarse como un requerimiento imprescindible de análisis previo, que nos permita adelantarnos a situaciones problemáticas como las descritas y que faciliten el diseño y establecimiento de momentos de formación y trabajo previos necesarios para acometer con éxito la experiencia.

Tampoco es comprensible el aislamiento dispuesto entre mundos virtuales. El intercambio y el trabajo colaborativo, compartir y relacionarse con diferentes personas no hace más que enriquecer y favorecer un aprendizaje más amplio y completo. Nos permite crecer, crear y dar a conocer conocimiento desde una plataforma más amplia de intercambio, aunque este aspecto no es asumido ni entendido por muchas de las experiencias, que abogan por mundos virtuales muy delimitados.

Es importante destacar la alta adecuación de los aspectos didácticos y pedagógicos. Se atiende en gran medida a aspectos instruccionales, de evaluación y seguimiento, respetando escrupulosamente parámetros tan importantes como son la sencillez, la accesibilidad, la coherencia de los recursos y la utilización de información sin ambigüedades, lo que permite afrontar el trabajo en mundos virtuales desde una buena perspectiva didáctica, adecuada y ajustada a los requerimientos propios.

No obstante, no es plausible que el trabajo en estas experiencias esté menguado por la utilización de escasos tipos de formatos, y aún más por la inexistencia de adaptaciones en función de los estilos de aprendizaje. Ofrecer y poder presentar la información en formatos variados debe ser un aspecto importante a considerar, ya que la carga visual y la exploración de éstos permite dotar a los alumnos de un papel más activo y participativo, asumiendo una amplia diversidad de interacciones y potenciando en mayor medida el interés por el descubrimiento. Por su parte, la escasez de adaptaciones limita el éxito de una experiencia educativa, siendo más pronunciada si nos referimos a entornos virtuales. Se ha de ser consecuente y abordar este tipo de procesos desde la atención hacia la diversidad.

Por último, incidir en la importancia del conocimiento de las características de los participantes. En gran medida no es viable ni oportuno implementar desarrollos educativos sin la consecución de un análisis inicial, que permita conocer aspectos tan básicos como son los conocimientos que disponen de los mundos virtuales, de su familiarización y actitud hacia las TIC, sus competencias comunicativas o saber de su grado de socialización. No se pueden plantear este tipo de experiencias mediante la equiparación de participantes y docentes por encontrarse en un mismo nivel educativo o disponer de edades similares. Es preciso y necesario profundizar más en las características propias de cada uno, en especial, en aquellos parámetros que van a ser necesarios e imprescindibles para que puedan trabajar y realizar una labor ajustada al nuevo entorno de trabajo. 
La importancia de este tipo de experiencias radica en su orientación hacia múltiples aprendizajes, lo que va favorecer en mayor grado la formación, la capacitación y la consecución de determinadas habilidades. Es importante el aprendizaje centrado en el alumno, al que todas las experiencias se orientan, así como el aprendizaje individual y colaborativo. Esta diversidad de aprendizajes vienen definidos por múltiples estrategias que se utilizan, por la secuenciación de contenidos, el establecimiento definido de entornos de trabajo, así como por la utilización de un avatar que permite una mayor identificación por parte de los participantes, una corporeidad definible en la mayoría de los casos que ayuda a la implicación y toma de papeles por parte de los alumnos en su formación.

\section{REFERENCIAS BIBLIOGRÁFICAS}

Arbeláez, M. (2010). Mundos Virtuales en la Educación en Salud. Simulación y aprendizaje en Open Simulator. (Tesis Doctoral, Universidad de Caldas). Recuperado de http:// www.maestriaendiseno.com/pdf/mauricioarbelaez.pdf.

Ausubel, D. P.; Novak, J. D.; Hanesian, H.; Sandoval Pineda, M. y Botero, M. (1976). Psicología Educativa: Un punto de vista cognoscitivo. México: Trillas.

Banet, M. (2001). Paradojas en los Entornos Virtuales. Santa Cruz, Chile: Ediciones País.

Cabero, J. (2006). Bases pedagógicas para la integración de las Tics en primaria y secundaria. Ponencia presentada en el II Congreso Internacional UNIVER. Tijuana, México. Resumen recuperado de http://tecnologiaedu.us.es/cuestionario/bibliovir/Bases456.pdf.

Cant, R. P. y Cooper, S. J. (2014). Simulation in the Internet age: The place of Web-based simulation in nursing education. An integrative review. Nurse Education Today, 34 (12), 1435-1442. doi: http://dx.doi.org/10.1016/j.nedt.2014.08.001.

Carr, D. (2008). Learning and Virtual World. En N. Selwyn (Ed.). Education 2.0? Designing the web for teaching and learning (pp. 13-17). Londres, Reino Unido: TLRP.

Carr, P. y Pond, G. (2007). Second Life: la guia definitiva a un nuevo mundo virtual. Barcelona, España: Random House Mondadori.

Castronova, E. (2001). Virtual Worlds: A First-Hand Account of Market and Society on the Cyberian Frontier. Indiana, EE. UU.: Cesifo Working Paper.

Cheal, C. (2007). Second Life: Hype or hyperlearning? On The Horizon, 15 (4), 204-210. Recuperado de http://www.emeraldinsight.com/doi/abs/10.1108/10748120710836228.

Cooper, H. M. (1989). Integrating Research: a guide for literature reviews. Beverly Hills, ca: Sage Publications.

Díaz Fernández, S. (2014). Desarrollo de una ficha de observación para el análisis y evaluación de experiencias educativas en mundos virtuales. IJERI: International Journal of Educational Research and Innovation, 1 (2), 69-82. Recuperado de https://www.upo. es/revistas/index.php/IJERI/article/view/1088/917.

Duart, J. M. (2003). Aprender sin distancias. Barcelona, España: UOc. Recuperado de http:// www.uoc.edu/web/esp/articles/josep_maria_duart.html.

Galagan, P. (2008). Second that! Recuperado de http://www.astd.org/Publications/Newsletters/Learning-Circuits/Learning-Circuits-Archives/2008/02/Second-That.

García Ruiz, M. A. (1998). Panorama General de las Aplicaciones de la Realidad Virtual en la Educación. Recuperado de http://www.sussex.ac.uk/informatics/.

Gibson, W. (1985). Neuromante. Canadá: Harper Voyager. 
Grané, M. y Muras, M. A. (2006). Second Life, entorno virtual, aprendizaje real. Comunicación y Pedagogía, 242-243, 49-56. Recuperado de http://161.116.23.180/repositori/ objectes/mg0001/sl_elearning.pdf.

Hew, K. F. y Cheung, W. S. (2010). Use of three-dimensional (3-D) immersive virtual worlds in K-12 and higher education settings: A review of the research. British Journal of Educational Technology, 41 (1), 33-55. doi:10.1111/j.1467-8535.2008.00900.x.

Huang, H.; Rauch, U. y Liaw, S. (2010). Investigating learners' attitudes toward virtual reality learning environments: Based on a constructivist approach. Computers \& Education, 55 (3), 1171-1182. doi:10.1016/j.compedu.2010.05.014.

Inman, C.; Wright, V. H. y Hartman, J. A. (2010). Use of Second Life in K-12 and Higher Education: A Review of Research. Journal of Interactive Online Learning, 9 (1), 44-63.

Kapp, K. M. (2007). Defining and Understanding Virtual Worlds. Recuperado de http:// www.astd.org/Publications/Newsletters/ASTD-Links/ASTD-Links-Articles/2007/04/ Defining-and-Understanding-Virtual-Worlds.

Kohler, T.; Fueller, J.; Stieger, D. y Matzler, K. (2010). Avatars - based innovation: Consequences of the virtual co-creation experience. Computer in human behavior, 27 (1), 160-168. doi: 10.1016/j.chb.2010.07.019.

Latiesa, M. (2003). Validez y fiabilidad de las observaciones sociológicas. En M. García Ferrando, J. Ibáñez y F. Alvira (Eds.). El análisis de la realidad social. Métodos y técnicas de investigación (pp. 409-443). Madrid, España: Alianza Editorial.

Leask, M. y Younie, S. (2001). Communal constructivist theory: information and communications technology pedagogy and internationalisation of the curriculum. Journal of Information Technology for Teacher Education, 10, 117-134. doi: 10.1080/14759390100200106.

Livingstone, D. y Kemp, J. (2006). Massively Multi-Learner: Recent Advances in 3D Social Environments. Computing and Information Systems Journal, 10 (2), 1-5. Recuperado de http://cis.uws.ac.uk/research/journal/v10n2/LinvingstoneKemp.doc.

Lizarralde, F. A. y Huapaya, C. R. (2012). Análisis de una plataforma virtual 3D descentralizada para el desarrollo de simulaciones educativas. Formación universitaria, 5 (6), 3-12. doi: 10.4067/S0718-50062012000600002.

Martínez, F. (2007). La integración escolar de las tecnologías educativas. En J. Cabero (Coord.). Nuevas Tecnologías Aplicadas a la Educación (pp. 22-40). Madrid, España: McGraw Hill.

Martínez, R. (2009). Mundos Virtuales 3D. Una guia para padres y formadores. Barcelona, España: Editorial UOC.

Mas, F. y Marín, B. (2008). Los metaversos en educación: el caso de Second Life y nuestra experiencia en formación. Quaderns digitals. Recuperado de http://www.quadernsdigitals.net/index.php?accionMenu=hemeroteca.DescargaArticuloIU.descarga\&tipo=PDF\&articulo_id=10479.

McMillan, J. H. y Schumacher, S. (2009). Research in education: evidence-based inquiry. Virginia, EE. UU.: Pearson Education.

Miller, M. y Jensen, R. L. (2014). Avatars in nursing: An integrative review. Nurse Educator, 39 (1), 38-41. doi: 10.1097/01.NNE.0000437367.03842.63.

Peachey, A. (2007). Education in Second Life. Computer Society of India Communications, 30 (12), 13-15. Recuperado de http://www.open.ac.uk/opencetl/files/opencetl/ file/ecms/web-content/Peachey-A-2007-Education-in-Second-Life-CSI-Communications-Journal-of-Computer-Society-of-India-31-12.pdf. 
Poveda, M. A. y Thous, M. C. (2013). Mundos virtuales y avatares como nuevas formas educativas. Historia y Comunicación Social, 18, 469-479. doi: 10.5209/rev_HICS.2013. v18.44262.

Rahman, M. H. A.; Yahaya, N. y Halim, N. D. A. (2014). Virtual World For Collaborative Learning: A Review. Documento presentado en la 2014 International Conference on Teaching and Learning in Computing and Engineering, EE. UU. doi: 10.1109/ LaTiCE.2014.18.

Ríos, J. y Ruiz, J. (2011). Competencias, Tic e innovación: Nuevos escenarios para nuevos retos. Revista de Docencia Universitaria, 10 (2), 467-470. Recuperado de http://dialnet. unirioja.es/descarga/articulo/4021144.pdf.

Rosenthal, R. (1991). Meta-analytic procedures for social research. Newbury Park, CA: Sage Publications.

Rutherfoord, R. H. y Rutherfoord, J. K. (2007). Universal instructional design for learning how to apply in a virtual world. Documento presentado en la VIII Conferencia ACM SIGITE de Tecnología de la Información en Educación, EE.UU. doi: 10.1145/1324302.1324332.

Sánchez, J. y Alto, M. (1989). Meta-análisis: una alternativa metodológica a las revisiones tradicionales de la investigación. En J. Arnau y H. Carpintero (Eds.). Tratado de psicología general I: historia, teoría y método (pp. 617-669). Madrid, España: Alhambra.

Sangrà, A. (2001). Enseñar y aprender en la virtualidad. Educar, 28, 117-131. Recuperado de http://educar.uab.cat/article/view/394/367.

Sherman, B. y Judkins, P. (1994). Glimpses of heaven, visions of hell: virtual reality and its applications. Londres, Reino Unido: Hodder \& Stoughton.

Smart, J.; Cascio, J.; Paffendorf, J.; Bridges; C., Hummel, J.; Hursthouse, J. y Moss, R. (2007). Pathways to the $3 D$ Web. A cross-industry public foresight project. Recuperado de http:// www.metaverseroadmap.org/MetaverseRoadmapOverview.pdf.

Stephenson, N. (1992). Snow crash. Nueva York, EE. UU.: Bantam Dell.

Urbina, A. B. y Flores, L. (2010). Mundo Virtual Educativo como apoyo al proceso de enseñanza-aprendizaje a nivel universitario. Documento presentado en el I Congreso Iberoamericano sobre Calidad de la Formación Virtual-CAFVIR 2010 (pp. 31-38). Alcalá de Henares, España. Resumen recuperado de http://www.esvial.org/cafvir2013/documentos/LibroActasCAFVIR2010.pdf.

Varela, G. A. (2010). Mundos virtuales educativos: una estrategia de aprendizaje para nativos digitales. Recuperado de http://148.202.167.76/igcaav/sites/default/files/capitulo\%20MV\%20gavn.pdf.

Wang, F. y Burton, J. K. (2012). Second Life in education: A review of publications from its launch to 2011. British Journal of Educational Technology, 44 (3), 357-371. doi: 10.1111/j.1467-8535.2012.01334.x.

Warburton, S. (2009). Second Life in higher education: Assessing the potential for and the barriers to deploying virtual worlds in learning and teaching. British Journal of Educational Technology, 40 (3), 414-426. doi: 10.1111 / j.1467-8535.2009.00952.x.

Warden, C. A.; Stanworth, J. O.; Biao, J. y Warden, A. R. (2013). Synchronous learning best practices: An action research study. Computers \& Education, 63, 197-207. doi: 10.1016/j. compedu.2012.11.010.

Wiersma, L. D. (2001). Conceptualization and development of the sources of enjoyment in youth sport questionnaire. Measurement in Physical Education and Exercise Science, 5 (3), 153-177. doi: 10.1207/S15327841MPEE0503_3. 